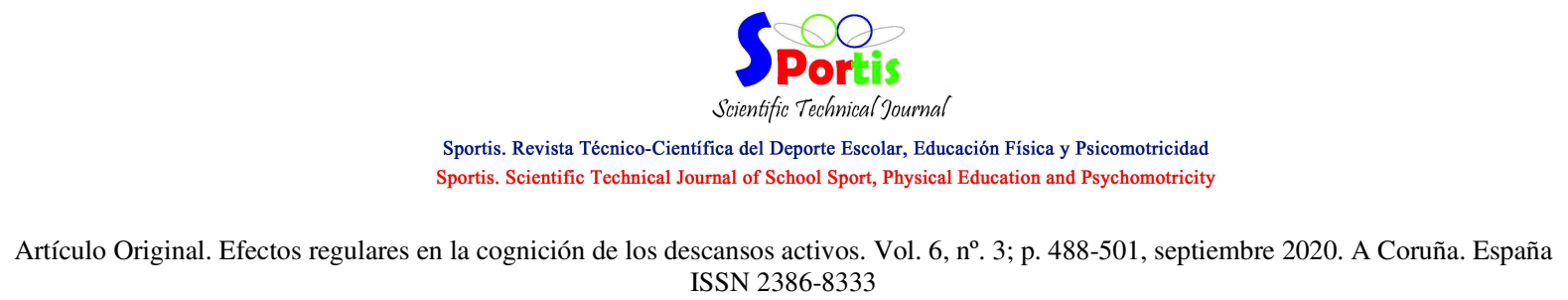
ISSN 2386-8333

\title{
Efectos regulares en la cognición de los descansos activos
}

\section{Chronic effects in cognition of actives-breaks}

\author{
Francisco Tomás González Fernández ${ }^{1,2,3}$; Salvador Baena Morales ${ }^{2,4}$; Moisés Vila Blanch ${ }^{1}$ \\ \& Olalla García-Taibo ${ }^{1}$ \\ ${ }^{1}$ Universidad Pontificia de Comillas (CESAG - Palma de Mallorca) \\ ${ }^{2}$ Universidad Internacional de Valencia \\ ${ }^{3}$ Universidad de Málaga \\ ${ }^{4}$ Departamento de Didácticas Generales y Didácticas Específicas. Universidad de Alicante
}

Contacto: salvador.baena@ua.es

Cronograma editorial: Artículo recibido: 25/04/2020 Aceptado: 13/07/2020 Publicado: 01/09/2020

DOI: https://doi.org/10.17979/sportis.2020.6.3.6414

\section{Resumen}

En el presente artículo se investigaron los efectos de un programa de entrenamiento físico de una duración de ocho semanas sobre la cognición del alumnado de $1^{\circ}$ de Bachillerato. Para lograr nuestro objetivo, dispusimos de dos grupos (Grupo control Vs Grupo experimental). Mientras, que el grupo control no realizó el entrenamiento, el grupo experimental realizó dos descansos activos a lo largo de una jornada escolar ( $1^{\circ}$ A las 10:00 a.m. y $2^{\circ}$ A las 12:30 p.m.). Antes de comenzar la intervención, en la primera semana, se tomaron los datos ambos grupos (pre). Se usó un diseño intra-sujeto con los factores condición de grupo. Los tiempos de reacción fueron medidos a través de un ANOVA de medidas repetida. La percepción del esfuerzo $\mathrm{s}$ datos fueron analizados realizando pruebas T-Student corregido por una prueba de Bonferroni para comparaciones múltiples. La intervención demostró su eficacia a nivel cognitivo, concretamente en atención sostenida. Por tanto, los resultados fueron coincidentes con la mayor parte investigaciones encontradas en la literatura sobre ejercicio físico regular y efectos positivos sobre las funciones cognitivas.

Palabras clave: entrenamiento; descansos activos; cognición; vigilancia

Para citar este artículo utilice la siguiente referencia: González Fernández, F.T.; Baena Morales, S.; Vila Blanch, M.; García-Taibo, O (2020). Efectos regulares en la cognición de los descansos activos. Sportis Sci J, 6 (3), 488-502.

DOI: https://doi.org/10.17979/sportis.2020.6.3.6414

http://revistas.udc.es/ 


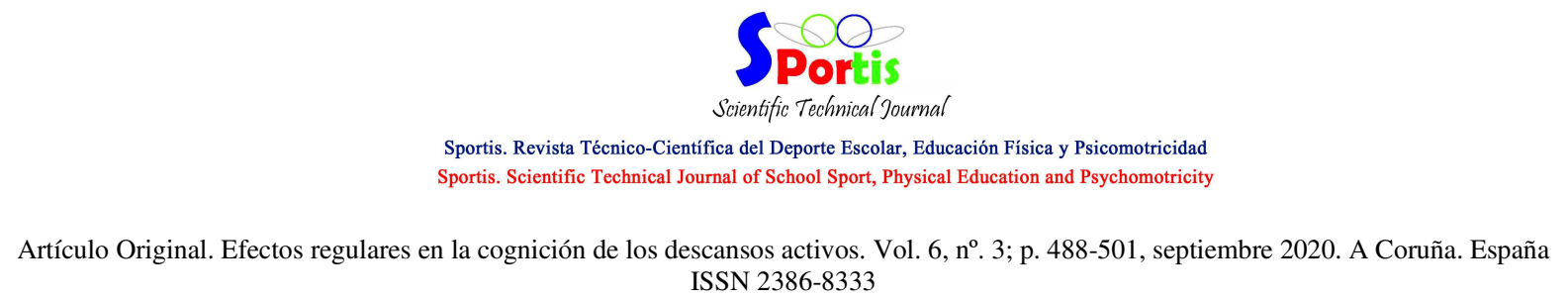
ISSN 2386-8333

\begin{abstract}
The present paper investigated the effect of a physical training program of 8-weeks on cognition of students of firs of high school to achieve or goal, we work with two groups (Control Group Vs Experimental Group). Control group did not to the intervention, the experimental group performed two active breaks during the school day (1 at 10:00 a.m. and 2 12:30 a.m.). Before to start the intervention, in first week, both groups (pre-intervention) data were registered. An intra-subject design with group condition factors was used. The reaction times were measured by a repeated measurement ANOVA. Perception of effort data was analyzed by performing T-Student tests corrected by a Bonferroni test for multiple comparisons. The intervention demonstrated its effectiveness at cognitive level, concretely in sustained attention. Therefore, results were consistent with most research found in the literature on regular physical exercise and positive effects on cognitive functions.
\end{abstract}

Keywords: training; active-breaks; cognition; vigilance

\title{
Introducción
}

Los beneficios que la actividad física tiene sobre la salud de los adolescentes han sido contrastados a lo largo de múltiples investigaciones (Ardoy et al., 2011; Martinez-Gomez et al., 2020). Dichos beneficios poseen un carácter preventivo y principalmente, son fundamentales para el desarrollo motriz y saludable en el alumnado de educación secundaria obligatoria (Rodriguez-Ayllon et al., 2019). Sin embargo, la mayoría de los efectos del ejercicio físico han sido confirmados sobre todo a nivel corporal (fisiológico), y en menor medida, sobre diferentes aspectos psicológicos (estrés, ansiedad, depresión, etc.). En este sentido, encontramos investigaciones sobre este importante tema de investigación, que buscan una relación inversa entre la práctica de actividad física y la aparición de depresión y estrés (Dunn et al., 2001) o ansiedad (Paluska \& Schwenk, 2000). Sin embargo, aunque son bien conocidos los beneficios de la actividad física comentados anteriormente, existe una incipiente tendencia (desde el año 2008 aproximadamente) que relaciona una mejora de las capacidades cognitivas (sobre todo sobre las funciones cognitivas de alto nivel), conexas con el rendimiento académico, y el ejercicio físico (Álvarez-Bueno et al., 2017; Daly-Smith et al., 2018; Donnelly et al., 2016). Por todo ello, nuestro interés en esta investigación versa sobre los efectos del ejercicio físico (descansos activos) realizados de forma regular en las funciones cognitivos.

Para citar este artículo utilice la siguiente referencia: González Fernández, F.T.; Baena Morales, S.; Vila Blanch, M.; García-Taibo, O (2020). Efectos regulares en la cognición de los descansos activos. Sportis Sci J, 6 (3), 488-502.

DOI: https://doi.org/10.17979/sportis.2020.6.3.6414

http://revistas.udc.es/ 


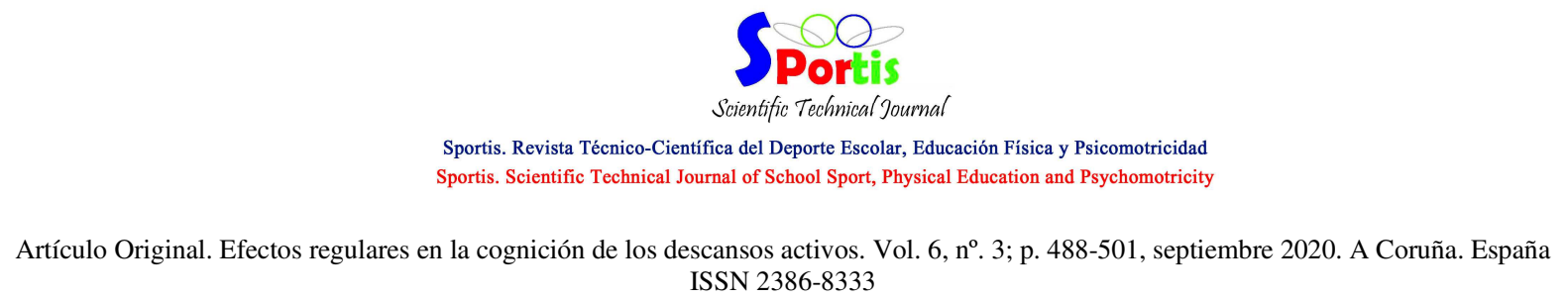
ISSN 2386-8333

Multitud de estudios han informado acerca de las bases teóricas de la neurociencia (Hötting \& Röder, 2013). Concretamente, sobre la atención, que como bien es sabido es una función cognitiva de alto nivel desde la que se inician en gran medida los procesos de memorización y consecuentemente del aprendizaje. Por todo ello, cuando hablamos de atención, debemos focalizarnos en un mecanismo cognitivo de alto nivel denominado "vigilancia". Así, la presente investigación, se centra en esta función cognitiva, que se encarga de proporcionar respuestas rápidas y precisas a estímulos relevantes que se presentan a lo largo de tareas de larga duración (Sarter, Givens \& Bruno, 2001). Contextualizando la presente investigación dentro del entorno académico, podríamos decir que un descenso en vigilancia en el contexto educativo lleva asociada un descenso del rendimiento cognitivo. De esta forma, es esencial determinar, dentro de un contexto educativo, cual es la génesis de la pérdida en vigilancia, ya que el origen de esta puede ser debido por una carencia o insuficiencia en cuanto a la orientación, selección y mantenimiento de la atención (Klimenko, 2011)

En la actualidad, muchos de los investigadores han mostrado un creciente interés existentes en el tópico de investigación que vincula el cerebro/cognición y el deporte/ejercicio físico (Walsh, 2014). De hecho, parece que la evidencia científica apunta hacia el importante rol que el ejercicio físico y el nivel de condición cardiovascular parecen ejercer sobre el rendimiento en contextos implicando a la vigilancia. No obstante, antes de hablar de esto, sería necesario clarificar todo lo relacionado con respecto a los efectos del ejercicio físico sobre el funcionamiento cognitivo, ya que principalmente estos se analizan desde dos perspectivas diferentes: 1) Teniendo en cuenta el ejercicio físico desde el punto de vista puntual [con efectos reversibles a corto plazo sobre el sistema cognitivo (ver Tomporowski, 2009 para una revisión)]. 2) Analizando los efectos el ejercicio físico sobre el sistema cognitivo con una práctica más regular [con efectos más duraderos sobre diferentes estructuras y funciones cerebrales (Colcombe y Kramer, 2003; Erickson et al., 2011)]. Además, Head \& Helton (2015) destacan como las tareas dinámicas que emplean nuevos

Para citar este artículo utilice la siguiente referencia: González Fernández, F.T.; Baena Morales, S.; Vila Blanch, M.; García-Taibo, O (2020). Efectos regulares en la cognición de los descansos activos. Sportis Sci J, 6 (3), 488-502.

DOI: https://doi.org/10.17979/sportis.2020.6.3.6414

http://revistas.udc.es/ 


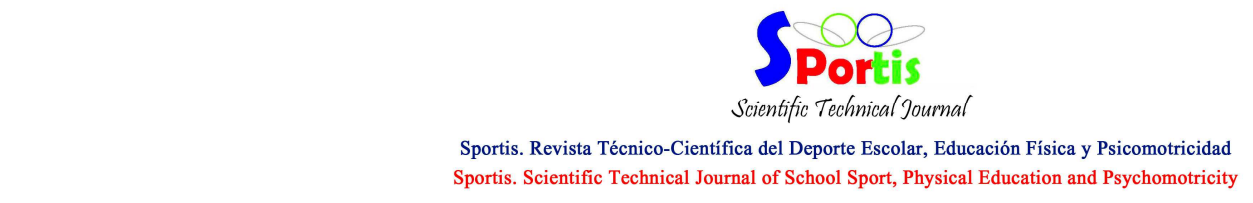

Artículo Original. Efectos regulares en la cognición de los descansos activos. Vol. 6, nº 3; p. 488-501, septiembre 2020. A Coruña. España ISSN 2386-8333

estímulos pueden dar como resultado la mezcla de diferentes procesos cognitivos, como la búsqueda activa de atención sostenida y aprendizaje perceptivo pasivo.

Sin embargo, existe cierta controversia respecto a los beneficios cognitivos de los descansos activos (Masini et al., 2020). Por ejemplo, Wilson et al., (2016) señala como los descansos activos son una herramienta efectiva para acumular actividad física moderada y vigorosa a lo largo de la jornada lectiva. Además, se ha evidenciado como una programación de descanso activos, puede llevar a una mejora del comportamiento y de la vitalidad subjetiva (Mavilidi et al., 2020). En relación con esta idea Layne et al., (2020) no encontró ningún efecto en el rendimiento matemático tras la realización de descansos activos. Sin embargo, señala como si podría intuirse una mejora cognitiva gracias a una intervención crónica. Un reciente Meta-análisis encuentra efectos positivos en el aumento de la actividad física a la hora de programar descansos activos, además de una mejora en el comportamiento en el aula. Sin embargo, destaca la aparición de resultados contradictorios para las funciones cognitivas y la mejora en el rendimiento académico (Masini et al., 2020).

Llegados a este punto, es fundamental realizar investigaciones que permitan analizar cuáles son las vías que se pueden implementar en un contexto educativo para la mejora de la vigilancia. Destacables aquí, son los estudios que hacen referencia a estudio a la relación entre la actividad física y los mecanismos atencionales del participante (Pontifex, Hillman \& Polich, 2009), destacando también numerosos trabajos que muestran una relación causal entre el nivel de actividad física y el rendimiento académico (Donnelly \& Lambourne, 2011;Janssen \& Leblanc, 2015)y a su vez, los trabajos que se relacionan con la adherencia a la actividad física (Ruiz-Ariza, A., et al. 2016). Esta asociación positiva entre el rendimiento cognitivo y la actividad física quedaría en parte explicada por una facilitación en la plasticidad de las sinapsis neuronales, así como el aumento del flujo sanguíneo en el cerebro (Pareja-Galeano y cols., 2013). Sin embargo, hasta donde alcanza nuestro conocimiento, no existen demasiados estudios que vinculen la relación entre la vigilancia y las anteriores variables mencionadas (dentro de un contexto educativo en educación secundaria obligatoria) por lo que es necesario realizar más investigación sobre como focalizar o activar al alumnado

Para citar este artículo utilice la siguiente referencia: González Fernández, F.T.; Baena Morales, S.; Vila Blanch, M.; García-Taibo, O (2020). Efectos regulares en la cognición de los descansos activos. Sportis Sci J, 6 (3), 488-502.

DOI: https://doi.org/10.17979/sportis.2020.6.3.6414

http://revistas.udc.es/ 

ISSN 2386-8333

durante la jornada escolar, mejorando sus funciones cognitivas y de forma complementaria su rendimiento académico.

Por lo tanto, el presente trabajo tiene como objetivo investigar el efecto de un programa de entrenamiento basado en descansos activos, sobre las funciones cognitivas, concretamente el rendimiento en la vigilancia, de un grupo de alumnos de $1^{\circ}$ de Bachillerato. Para alcanzar el anterior objetivo, se medirán variables tales como son la velocidad de respuesta ante un estímulo visual, usando una tarea clásica de vigilancia [Psycomotor Vigilance Task (PVT)], considerada como una tarea fundamental para valorar el rendimiento de los participantes (González, Oyarzo, Fischer, De la Fuente, Diaz, \& Berral, 2011) Esta variable está relacionada con la vigilancia, entendida como una función cognitiva superior que determina la predisposición de responder a un estímulo relevante y asignar con efectividad los recursos atencionales en el tiempo como sucede en el ámbito académico.
\end{abstract}

\title{
Metodología
}

\section{Participantes.}

Un total de 30 alumnos de $1^{\circ}$ de Bachillerato (años: $16.42 \pm 0.34$; altura: $174.04 \pm$ 8.15; peso: $64.45 \pm 11.53$ e IMC: $22.49 \pm 3.17$ ) participaron en la presente investigación. Todos los participantes completaron un cuestionario general de hábitos de vida saludables y el cuestionario internacional de actividad física en versión corta (IPAQ-SF; Lee et al., 2011). En el primero, se les preguntaba sobre hábitos deportivos actuales, adicciones y enfermedades que pudieran impedir la práctica de ejercicio físico, mientras que, en el segundo, podíamos observar el nivel de actividad física de los participantes. Los datos del cuestionario y las preguntas sobre hábitos de vida de los participantes nos mostraron el nivel de actividad física "moderado" (Mets= $709.45 \pm 187.88)$ y que estos no tenían ninguna lesión parcial/crónica, ni sufrían ningún trastorno neurológico que pudiera. Todos los participantes fueron informados acerca de los objetivos de la investigación y de los posibles riesgos de participar en ella. Además, el estudio se realizó de acuerdo con las guías éticas de investigación humana y bajo las normas establecidas en Declaración de Helsinki de 1964.

\section{Aparatos y material}

Para citar este artículo utilice la siguiente referencia: González Fernández, F.T.; Baena Morales, S.; Vila Blanch, M.; García-Taibo, O (2020). Efectos regulares en la cognición de los descansos activos. Sportis Sci J, 6 (3), 488-502. 


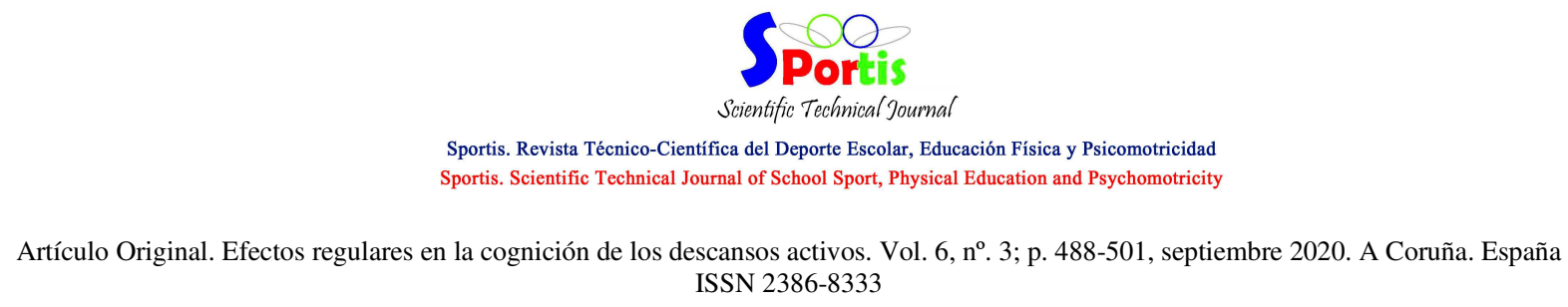

Se usó la escala de Borg (Borg, 1982) para registrar la percepción subjetiva del esfuerzo a PSE, que posee un rango de percepción del esfuerzo que va desde 6 (muy ligero) a 20 (muy muy duro). Los participantes tenían experiencia con esta escala y se utilizó para cerciorarnos que la intensidad de la sesión cumplía con las recomendaciones del ACSM (2010). Los datos de percepción subjetiva del esfuerzo (PSE) fueron controlados inmediatamente después de las sesiones de descansos activos. según la escala de Borg. La PSE fue registrada mediante la escala de Borg (Borg, 1982) que posee un rango de percepción del esfuerzo que va desde 6 (muy ligero) a 20 (muy muy duro).

Para presentar los estímulos de la PVT, se utilizaron diferentes dispositivos móviles [Apple by California (IOS, versión 13.3.1). El centro de la pantalla del dispositivo móvil se encontraba situado entre 60 y $80 \mathrm{~cm}$ de la cabeza de los participantes y al nivel de sus ojos. La aplicación móvil Vigilance Buddy (Michael Rademaker) se utilizó para controlar la presentación del estímulo y la recogida de datos. Las respuestas de los participantes fueron recogidas al pulsar la pantalla del dispositivo (Ver Figura 1). La PVT consistió en la presentación de unos números en el centro de la pantalla, que comenzaron a completarse a la velocidad de un cronómetro real y que se podían presentar en la pantalla después de un intervalo de tiempo aleatorio que oscilaba entre 2000 y $10000 \mathrm{~ms}$. Antes del inicio de la PVT en ambas sesiones, se proporcionaron instrucciones verbales a los participantes, haciendo hincapié en que había que fijar la vista en el centro de la pantalla, tratando de no mover los ojos y responder lo más rápido posible evitando anticipaciones. La tarea comprendía un solo bloque de duración de 10 minutos

Para citar este artículo utilice la siguiente referencia: González Fernández, F.T.; Baena Morales, S.; Vila Blanch, M.; García-Taibo, O (2020). Efectos regulares en la cognición de los descansos activos. Sportis Sci J, 6 (3), 488-502.

DOI: https://doi.org/10.17979/sportis.2020.6.3.6414

http://revistas.udc.es/ 


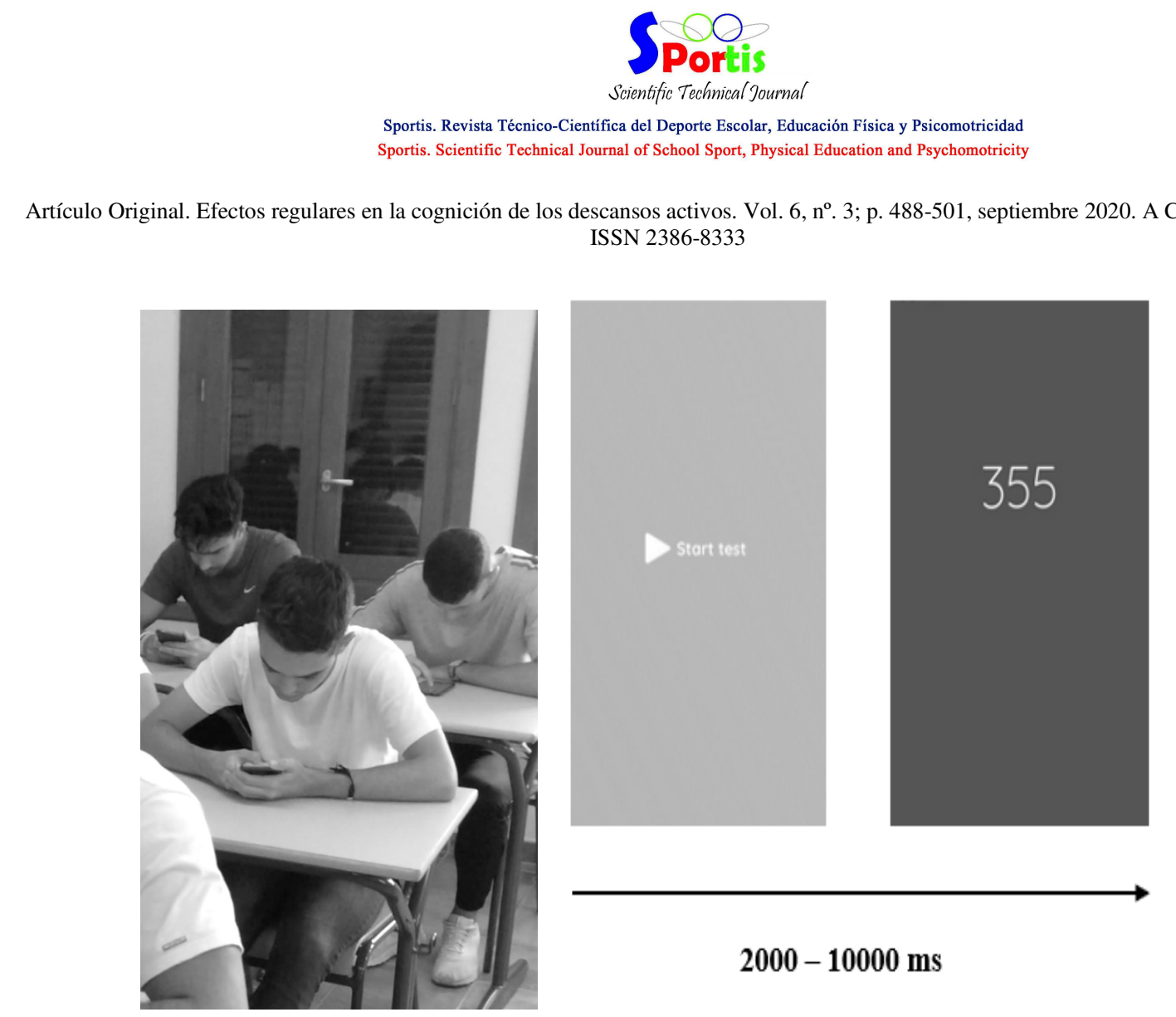

Figura 1. Ejemplo de un ensayo de la tarea de vigilancia psicomotora y participante realizándola.

Los participantes fueron instruidos para la realización de la tarea con anterioridad, remarcando que debían hacer clic en la pantalla tan rápido como les fuese posible al iniciar el contador de tiempo. Previamente a la toma de datos, cada alumno pudo emplear varios intentos con la finalidad de familiarizarse con la aplicación. Esto no supuso ningún problema en cuanto a efecto de aprendizaje por el carácter aleatorio secuencial de la tarea de vigilancia. En primer lugar, se les instruyó a los alumnos en relación con el software y proceso de evaluación. Seguidamente se realizó una evaluación inicial por medio de dos pruebas, una para determinar el nivel de atención sostenida y otra para normalizar el nivel de esfuerzo del sujeto pre-intervención, utilizando la escala de percepción del esfuerzo de Börg (Börg, 1998) en la cual fueron instruidos con anterioridad. Ambos grupos realizaron la primera recogida de datos en el gimnasio del centro, aplicando la misma duración a cada registro (en grupos de 5 personas). Tras esto, para el grupo experimental, se les instruyó en el programa de descansos activos. De nuevo, tras pasar ochos semanas, ambos grupos fueron evaluados de nuevo, en condiciones y forma idénticas a la evaluación inicial.

Para citar este artículo utilice la siguiente referencia: González Fernández, F.T.; Baena Morales, S.; Vila Blanch, M.; García-Taibo, O (2020). Efectos regulares en la cognición de los descansos activos. Sportis Sci J, 6 (3), 488-502.

DOI: https://doi.org/10.17979/sportis.2020.6.3.6414

http://revistas.udc.es/ 


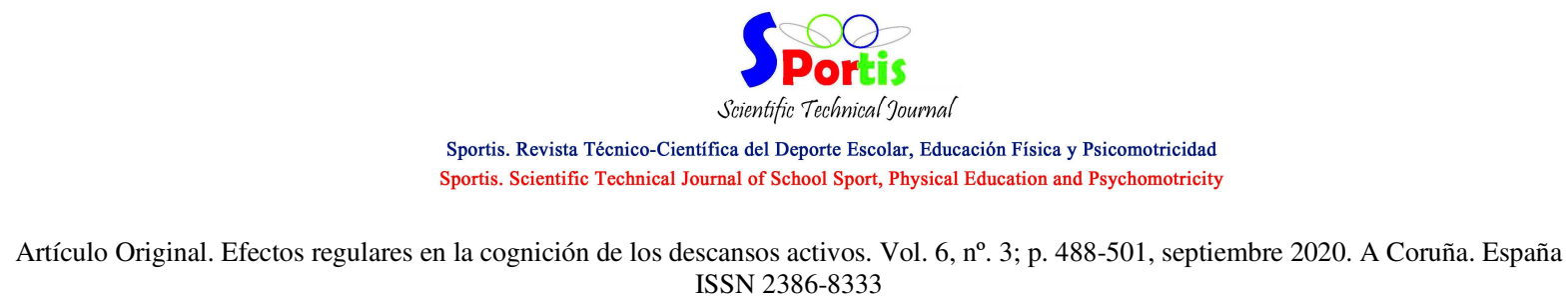
ISSN 2386-8333

Para controlar que los cuestionarios de Google. En ello, el experimentador observaba la evolución personal de las participantes. Además, al final de cada semana de descanso les llegaba un correo al cual debían responder para valorar la intensidad de la intervención se estaba cumpliendo correctamente.

Suarez-Manzano, Ruiz-Ariza, Lopez-Serrano, \& López, (2018) a través de una revisión sobre los DA hallaron un rango de mejoras al introducir secuencias de Actividad Física a intensidad Moderada-Vigorosa (AFMV) de 5min de duración frente a 30 min de duración con menos intensidad. Los ejercicios dispuestos en la tabla anterior fueron adaptados para aquellos alumnos con alguna limitación o dificultad en el momento de la intervención.

Análisis estadístico

Se utilizó un diseño intra-sujeto con los factores condición de grupo [grupo control (GC) y grupo experimental (GE)]. Un ANOVA de medidas repetidas fue utilizado para analizar los RTs de los grupos. Los datos de PSE fueron analizados realizando pruebas TStudent corregido por una prueba de Bonferroni para comparaciones múltiples para la comparación de medias en grupos semejantes en dos momentos determinados de la intervención (pre y post). El tamaño del efecto es partial eta squared para las Fs.

\section{Resultados}

\section{Tarea de Vigilancia Psicomotora}

Un ANOVA con la media de los TR de los participantes de Pre (GC y GE), no reveló ningún efecto, $F(1,19)=2.91, p<.10, \eta^{2}$ partial=.13, demostrando que los grupos en la preintervención eran iguales y no había existían diferencias previas, grupo control en la fase pre $(375.40 \pm 108)$ experimental $(371.56 \pm 105)$.

Tras la intervención, un análisis similar se realizó de nuevo para los TRs del Post (GC y GE), los datos revelaron un efecto principal de fase, $F(1,19)=6.39, p<.001, \eta^{2}$ partial=.25. Los participantes del grupo experimental respondieron más rápido que los del grupo control en la fase post $(378.70 \pm 126)$ experimental $(352.03 \pm 115)$. Por lo tanto, el grupo que realizó el programa de entrenamiento basado en los descansos activos obtuvo mejores puntuaciones reduciendo el tiempo medio de respuesta en la tarea PVT. (Ver tabla 1 para más información)

Para citar este artículo utilice la siguiente referencia: González Fernández, F.T.; Baena Morales, S.; Vila Blanch, M.; García-Taibo, O (2020). Efectos regulares en la cognición de los descansos activos. Sportis Sci J, 6 (3), 488-502. 


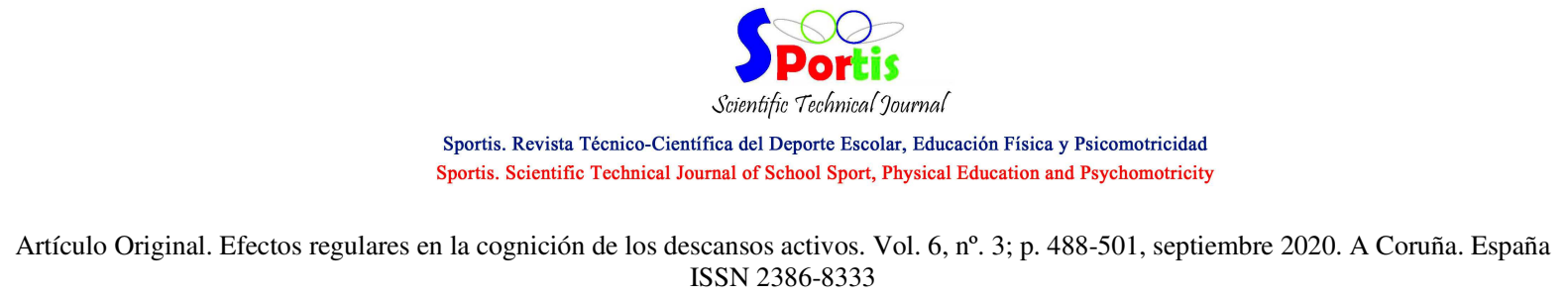
ISSN 2386-8333

Tabla 1. Medias de ambos grupos tras realizar el programa de actividad física. análisis estadísticos TVP (ms).

\begin{tabular}{cccc}
\hline \multicolumn{2}{c}{ Pre } & \multicolumn{2}{c}{ Post } \\
\hline GC & GE & GC & GE \\
\hline $375.40 \pm 108$ & $371.56 \pm 108^{*}$ & $378.70 \pm 126$ & $352.03 \pm 115^{*}$ \\
\hline
\end{tabular}

\section{Discusión}

La presente investigación tiene como objetivo analizar los efectos crónicos de un programa ejercicio físico basado en los descansos activos sobre la vigilancia en un grupo de alumnos de $1^{\circ}$ de bachillerato. Los resultados del estudio muestran como los participantes que realizaron un entrenamiento combinado de ejercicio físico aeróbico y de fuerza mediante los descansos-activos fueron significativamente mejores en vigilancia tras la intervención que los que no realizaron descansos activos.

Uno de los objetivos de esta investigación era el de implementar una intervención de DA que tuviera cabida en la realidad educativa. De este modo, la propuesta presentada pudiera ser transferible a cualquier centro educativo sin que supongo un excesivo cambio en la rutina horaria. Para ello, además de las rutinas descritas en otros trabajos (Martínez-López et al. 2018) se ha tenido presente a la hora de seleccionar los ejercicios el utilizar tareas que el alumnado ya conociera o fueran fácil de dominar, por lo que la selección de tareas de corta duración pareció ser adecuada para la realización ente horas de clase. Además, para favorecer el valor educativo que se pretende, se realizaron ejercicios con una orientación de interacción directa entre sujetos, conllevando al mismo tiempo una mayor predisposición hacia la realización de la tarea (Franco, Coterón, Martínez, \& Brito, 2017). Esta idea es fundamental ya que la adherencia a la práctica de actividad física es una de las claves para potenciar los efectos en las capacidades cognitivas (Ruiz-Ariza, Ruiz, De La Torre-Cruz, Latorre-Román, \& Martínez-López, 2016). En cuanto a lo que involucra a la tarea de vigilancia, es preciso comentar que es una tarea muy sensible para medir vigilancia (Gunzelmann, Moore, Gluck,

Para citar este artículo utilice la siguiente referencia: González Fernández, F.T.; Baena Morales, S.; Vila Blanch, M.; García-Taibo, O (2020). Efectos regulares en la cognición de los descansos activos. Sportis Sci J, 6 (3), 488-502. 
Van Dongen, \& Dinges (2009) y que la aparición de diferencias significativas respecto a la vigilancia podría confirmar los resultados encontrados en otras investigaciones donde mostraron diferencias en vigilancia (Davies \& Parasuraman, 1983; Van Dongen \& Belenky, 2008). No obstante, hay que destacar que en la literatura no existe un conceso claro sobre qué cantidad y que intensidad de actividad física (dosis-respuesta) es la adecuada para producir un mayor efecto fisiológico sobe las funciones ejecutivas. Suárez-Manzano et al. (2017) a través de una revisión sobre los DA hallaron un rango de mejoras al introducir secuencias de Actividad Física a intensidad Moderada-Vigorosa (AFMV) de 5 min de duración frente a 30min de duración con menos intensidad. En la misma revisión se expuso como los grupos de control (sin intervención DA), de todos los artículos analizados, no mejoraron los resultados en variables cognitivas como atención, memoria y planificación. Este resultado coincide con los encontrados en nuestra investigación, fortaleciendo que la no intervención podría tener un efecto negativo en el grupo control. El número de participantes $(\mathrm{n}=)$ fue muy diverso, encontrando trabajos con $n=24$ (Donnelly \& Lambourne, 2011) a estudios como el formulado por Katz et al. (2010) con una $n=1214$.

\title{
Conclusión
}

Tras la realización de esta investigación podemos concluir que el ejercicio moderado aeróbico puede producir un aumento de la vigilancia psicomotora de los participantes, mejorando la eficacia en atención sostenida. Se puede sugerir que un programa de entrenamiento basado en descansos activos podría ayudar a mantener la concentración durante más tiempo y favorecer la absorción de conocimientos tras los mismos después de un periodo de actividad sedentaria. Otra línea interesante de estudio resultante sería establecer correlaciones entre la facilidad de aprendizaje de diferentes materias con estímulos fisiológicos específicos resultantes de la actividad física. De esta forma se establecen parte de las bases para la realización de posteriores investigaciones, desde las cuales ir definiendo la efectividad de diferentes variables, que, en conjunto, nos ayuden a diseñar un programa de intervención efectivo y sobre todo realista a las demandas curriculares del aula.

Para citar este artículo utilice la siguiente referencia: González Fernández, F.T.; Baena Morales, S.; Vila Blanch, M.; García-Taibo, O (2020). Efectos regulares en la cognición de los descansos activos. Sportis Sci J, 6 (3), 488-502.

DOI: https://doi.org/10.17979/sportis.2020.6.3.6414

http://revistas.udc.es/ 


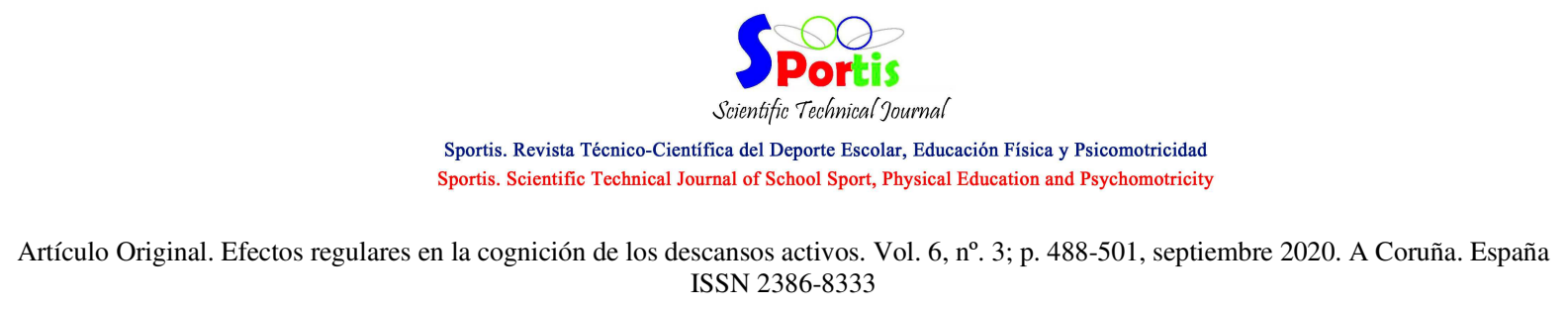

\section{Referencias}

Álvarez-Bueno, C., Pesce, C., Cavero-Redondo, I., Sánchez-López, M., Martínez-Hortelano, J. A., y Martínez-Vizcaíno, V. (2017). The Effect of Physical Exercise Activity Interventions on Children's Cognition and Metacognition: A Systematic Review and Meta-Analysis. Journal of the American Academy of Child \& Adolescent Psychiatry, 56(9), 279-738. DOI: https://doi.org/10.1016/j.jaac.2017.06.012

American College of Sports Medicine (2010). ACSM's guidelines for exercise testing and prescription. 9th. ed. London: Lippincott Williams \& Wilkins.

Ardoy, D. N., Fernández-Rodríguez, J. M., Ruiz, J. R., Chillón, P., España-Romero, V., Castillo, M. J., y Ortega, F. B. (2011). Improving Physical Fitness in Adolescents Through a School-Based Intervention: the EDUFIT Study. Revista Española de Cardiología (English Edition), 64(6), 484-491. DOI: https://doi.org/10.1016/j.rec.2011.02.010

Best, J. R. (2010). Effects of physical activity on children's executive function: contributions of experimental research on aerobic exercise. Developmental Review, 30, 331-551. DOI: https://doi.org/10.1016/j.dr.2010.08.001

Borg, G. (1998). Borg's perceived exertion and pain scales. Champaign, IL, US: Human Kinetics.

Chaddock, L., Erickson, K.I., Prakash, R.S., Voss, M.W., VanPatter, M., Pontifex, M.B., Hillman, C.H., y Kramer, A.F. (2012). A functional MRI investigation of the association between childhood aerobic fitness and neurocognitive control. Biological Psychology, 89(1):260-8. DOI: https://doi.org/10.1016/j.biopsycho.2011.10.017

Colcombe, S. J., Kramer, A. F., Erickson, K. I., Scalf, P., McAuley, E., y Cohen, N. J. (2004). Cardiovascular fitness, cortical plasticity, and aging. Proceedings National Academy Science, 101(9), 3316-3321. DOI: https://doi.org/10.1073/pnas.0400266101

Davies, R.D., y Parasuraman, R. (1982). The psychology of vigilance. Academic Press.

Daly-Smith, A. J., Zwolinsky, S., McKenna, J., Tomporowski, P. D., Defeyter, M. A., y Manley, A. (2018). Systematic review of acute physically active learning and

Para citar este artículo utilice la siguiente referencia: González Fernández, F.T.; Baena Morales, S.; Vila Blanch, M.; García-Taibo, O (2020). Efectos regulares en la cognición de los descansos activos. Sportis Sci J, 6 (3), 488-502. DOI: https://doi.org/10.17979/sportis.2020.6.3.6414 http://revistas.udc.es/ 


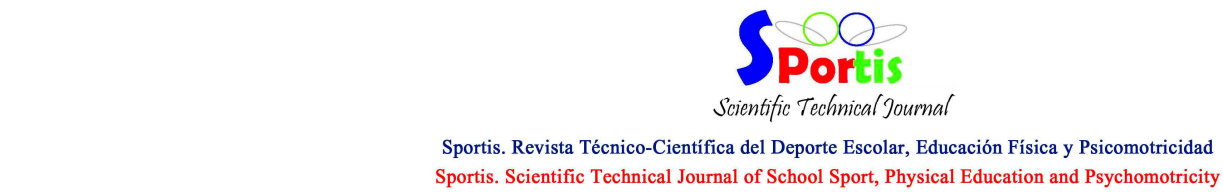

Artículo Original. Efectos regulares en la cognición de los descansos activos. Vol. 6, nº 3; p. 488-501, septiembre 2020. A Coruña. España ISSN 2386-8333

classroom movement breaks on children's physical activity, cognition, academic performance and classroom behaviour: Understanding critical design features. Open Sport and Exercise Medicine, 4(1), 1-16. DOI: https://doi.org/10.1136/bmjsem-2018000341

Donnelly, J. E., Hillman, C. H., Castelli, D., Etnier, J. L., Lee, S., Tomporowski, P., SzaboReed, A. N. (2016). Physical activity, fitness, cognitive function, and academic achievement in children: A systematic review. Medicine and Science in Sports and Exercise, 48(6), 1197-1222. DOI: https://doi.org/10.1249/MSS.0000000000000901

Donnelly, J. E., y Lambourne, K. (2011). Classroom-based physical activity, cognition, and academic achievement. Preventive Medicine, 52(SUPPL.), S36-S42. DOI: https://doi.org/10.1016/j.ypmed.2011.01.021

Dunn, M.E., Burbine, T., Bowers, C.A., Tantleff-Dunn, S. (2001). Moderators of stress in parents of children with autism. Community Mental Health Journal, 37(1):39-52. DOI: https://doi.org/10.1023/A:1026592305436

Erickson, K. I., Voss, M. W., Prakash, R. S., Basak, C., Szabo, A., Chaddock, L., Kramer, A. F. (2011). Exercise training increases size of hippocampus and improves memory. Proceeding of the National Academy of Sciences of the United States of America, 108(7), 3017-3022. DOI: https://doi.org/10.1073/pnas.1015950108

Franco, E., Coterón, J., Martínez, H. A., y Brito, J. (2017). Perfiles motivacionales en estudiantes de educación física de tres países y su relación con la actividad física. Suma Psicológica, 24(1), 1-8. DOI: https://doi.org/10.1016/j.sumpsi.2016.07.001

Gunzelmann, G., Moore, L.R., Martin, L., Gluck, K.A., Dongen, H.P., \& Dinges, D.F. (2010). Fatigue in Sustained Attention: Generalizing Mechanisms for Time Awake to Time on Task. DOI: https://doi.org/10.1037/12343-004

Head, J., y Helton, W. S. (2015). Passive perceptual learning versus active searching in a novel stimuli vigilance task. Experimental brain research, 233(5), 1481-1489. DOI: https://doi.org/10.1007/s00221-015-4222-z

Para citar este artículo utilice la siguiente referencia: González Fernández, F.T.; Baena Morales, S.; Vila Blanch, M.; García-Taibo, O (2020). Efectos regulares en la cognición de los descansos activos. Sportis Sci J, 6 (3), 488-502. DOI: https://doi.org/10.17979/sportis.2020.6.3.6414 http://revistas.udc.es/ 
Hötting, K., y Röder, B. (2013). Beneficial effects of physical exercise on neuroplasticity and cognition. Neuroscience and Biobehavioral Reviews, 37(9), 2243-2257. DOI: https://doi.org/10.1016/j.neubiorev.2013.04.005

Janssen, I., y Leblanc, A. (2015). Systematic Review of the Health Benefits of Physical Activity and Fitness in School-Aged Children and Youth. School Nutrition and Activity, 7(40) 183-219. DOI: https://doi.org/10.1201/b18227-14

Katz, D. L., Cushman, D., Reynolds, J., Njike, V., Treu, J. A., Walker, J., Katz, C. (2010). Putting physical activity where it fits in the school day: Preliminary results of the ABC (Activity Bursts in the Classroom) for fitness program. Preventing Chronic Disease, 7(4). Extraído de: http://www.cdc.gov/pcd/issues/2010/jul/09_0176.htm

Klimenko, O. (2011). Fomento de la capacidad creativa desde las prácticas de enseñanza. $\begin{array}{llll}\text { Revista de Filología } \quad \text { Hispánica, 7(12), 89-99. DOI: } & \end{array}$ https://doi.org/10.25057/21452776.803

Layne, T., Yli-Piipari, S., y Knox, T. (2020). Physical activity break program to improve elementary students' executive function and mathematics performance. Education 313, 0(0), 1-9. DOI: https://doi.org/10.1080/03004279.2020.1746820

Mavilidi, M. F., Mason, C., Leahy, A. A., Kennedy, S. G., Eather, N., Hillman, C. H., Morgan, P. J., Lonsdale, C., Wade, L., Riley, N., Heemskerk, C., y Lubans, D. R. (2020). Effect of a Time-Efficient Physical Activity Intervention on Senior School Students' On-Task Behaviour and Subjective Vitality: the 'Burn 2 Learn' Cluster Randomised Controlled Trial. Educational Psychology Review. DOI: https://doi.org/10.1007/s10648-020-09537-x

Paluska, S.A., y Schwenk, T.L. (2000). Physical activity and mental health: current concepts. Sports Medicine, 29(3):167-80. DOI: https://doi.org/10.2165/00007256-20002903000003

Pontifex, M.B, Scudder, M.R, Drollette, E.S., y Hillman, C.H. (2012). Fit and vigilant: the relationship between poorer aerobic fitness and failures in sustained attention during $\begin{array}{lll}\text { preadolescence. } & \text { Neuropsychology. }\end{array}$ https://doi.org/10.1037/a0028795

Para citar este artículo utilice la siguiente referencia: González Fernández, F.T.; Baena Morales, S.; Vila Blanch, M.; García-Taibo, O (2020). Efectos regulares en la cognición de los descansos activos. Sportis Sci J, 6 (3), 488-502. DOI: https://doi.org/10.17979/sportis.2020.6.3.6414

http://revistas.udc.es/ 


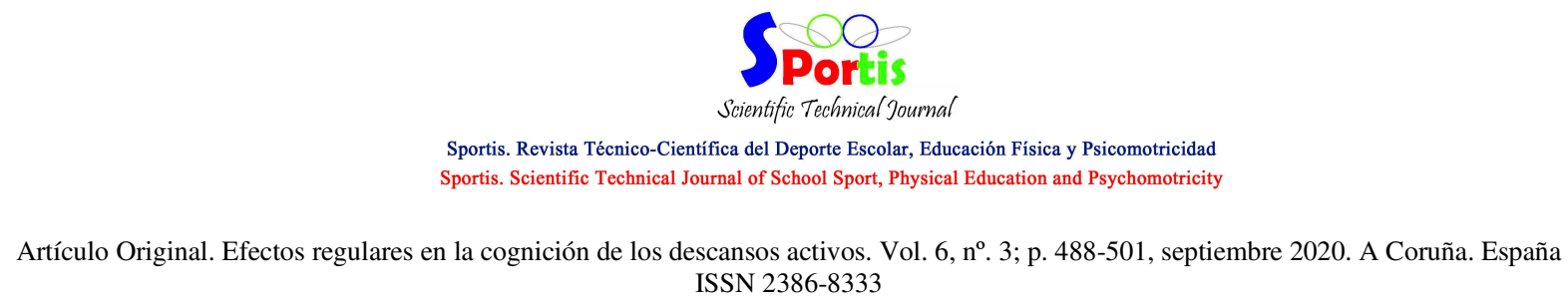

Rodriguez-Ayllon, M., Cadenas-Sánchez, C., Estévez-López, F., Muñoz, N. E., MoraGonzalez, J., Migueles, J. H., Esteban-Cornejo, I. (2019). Role of Physical Activity and Sedentary Behavior in the Mental Health of Preschoolers, Children and Adolescents: A Systematic Review and Meta-Analysis. Sports Medicine, 49(9), 13831410. DOI: https://doi.org/10.1007/s40279-019-01099-5

Ruiz-Ariza, A., Ruiz, J. R., De La Torre-Cruz, M., Latorre-Román, P., y Martínez-López, E. J. (2016). Influencia del nivel de atracción hacia la actividad física en el rendimiento académico de los adolescentes. Revista Latinoamericana de Psicología, 48(1), 42-50. DOI: https://doi.org/10.1016/j.rlp.2015.09.005

Sarter, M., Given, B., y Bruno, J.P. (2001). The cognitive neuroscience of sustained attention: where top-down meets bottom-up. Brain Research Reviews, 35(2):146-160. DOI: https://doi.org/10.1016/S0165-0173(01)00044-3

Suarez-Manzano, S., Ruiz-Ariza, A., López-Serrano, S., y López, E. J. M. (2018). Active breaks to improve class attention. Educational Interventions. Profesorado 4. DOI: https://doi.org/10.30827/profesorado.v22i4.8417

Tomporowski, P. D. (2009). Methodological issues: Research approaches, research design, and task selection. En T. McMorris, P. D. Tomporowski y M. Audiffren (Eds), Exercise and Cognitive Function (pp. 91-112). Chichester: John Wiley y Sons. DOI: https://doi.org/10.1002/9780470740668.ch4

Tomporowski, P. D., Lambourne, K., y Okumura, M. S. (2011). Physical activity interventions and children's mental function: an introduction and overview. Preventive Medicine, 52 (Suppl 1), S3-9. DOI: https://doi.org/10.1016/j.ypmed.2011.01.028

Van Dongen, E.V., Kersten, I.H., Wagner, I.C., Morris, R.G., y Fernández (2016). Physical Exercise Performed Four Hours after Learning Improves Memory Retention and Increases Hippocampal Pattern Similarity during Retrieval. Current Biology, 26 (13):1722-7. DOI: https://doi.org/10.1016/j.cub.2016.04.071

Walsh, V. (2014). Is sport the brain's biggest challenge? Current Biology, 24(18), R859R860. DOI: https://doi.org/10.1016/j.cub.2014.08.003

Para citar este artículo utilice la siguiente referencia: González Fernández, F.T.; Baena Morales, S.; Vila Blanch, M.; García-Taibo, O (2020). Efectos regulares en la cognición de los descansos activos. Sportis Sci J, 6 (3), 488-502. DOI: https://doi.org/10.17979/sportis.2020.6.3.6414 http://revistas.udc.es/ 
Artículo Original. Efectos regulares en la cognición de los descansos activos. Vol. 6, nº 3; p. 488-501, septiembre 2020. A Coruña. España ISSN 2386-8333

Wilson, A. N., Olds, T., Lushington, K., Petkov, J., y Dollman, J. (2016). The impact of 10minute activity breaks outside the classroom on male students' on-task behaviour and sustained attention: A randomised crossover design. Acta Paediatrica, International Journal of Paediatrics, 105(4), e181-e188. DOI: https://doi.org/10.1111/apa.13323 